\title{
Framework model on enterprise information system based on Internet of things
}

\section{Zhengxi Wei}

School of Computer Science, Sichuan University of Science \& Engineering, Zigong Sichuan 643000, PR China

\author{
Email address: \\ 413789256@qq.com
}

\section{To cite this article:}

Zhengxi Wei. Framework Model on Enterprise Information System Based on Internet of Things. International Journal of Intelligent Information Systems. Vol. 3, No. 6, 2014, pp. 55-59. doi: 10.11648/j.ijiis.20140306.11

\begin{abstract}
A high degree of information sharing can enable manufacturing and services industry to optimize business processes and improve the core competitiveness and innovation ability. This paper aims to explore a framework model to build a kind of management information system based on Internet of things. Starting from the demand and functions of the system, we firstly outline a conceptual model based on sensing networks. Next, we construct the systemic framework and illustrate its running procedure in detail. Finally, we give experimental schemes designed for each module under J2EE platform. Practical application proves that our system can display the important information of tracking object in real time and achieve a more efficient management. Based on this model, people can further research and establish custom rules used to general information processing, make manufacturing and services enterprises build a modern information system more efficiently and faster, and facilitate practical application for Internet of things technology.
\end{abstract}

Keywords: Intelligent System, RFID Middleware, Framework Design, Enterprise Information System, Conceptual Model

\section{Introduction}

With the rapid development of cloud computing and RFID middleware [1] technology, etc., they are integrated with manufacturing technology, forming the core technology during the informatization procedure of manufacturing and services industry, driving its informatization extent to a new level, and promoting its sustainable development. Smart manufacturing [2] and services supported by sensing networks, database technology, business intelligence, virtual simulation, modelling technology and so on, makes the product intelligence, manufacturing process automation, and provides applications for aided management decision.

Thus, the informatization procedure of manufacturing and services industry combines information technology, automation technology, modern management techniques with manufacturing technology, and becomes a system engineering that improves manufacturing and service industries integrity, sustainability and service ability. Internet of things [3] through comprehensive perception, reliable delivery and intelligent processing makes the information reach different destinations so as to achieve "things - things" connected and information sharing. Manufacturing and services industries are required to achieve the informatization management from product design and manufacturing to sales and service during the entire life cycle, while Internet of things technology can fitly help them gain this advantage.

Based on Internet of things technology, a high degree of information sharing enables enterprises to optimize business processes and resource allocation, strengthening detail management and process management, promotes that enterprises have to continuously adapt to changes in the external environment, and improves the core competitiveness and innovation ability. For example, Lockheed Martin military company, which uses advanced management information system (MIS), during the development of JSF aircraft, tooling reduces $90 \%$, production time reduces $66 \%$ and manufacturing costs reduce $50 \%$. This paper aims to explore and establish a framework model used to build MIS based on Internet of things. Based on this model, people can further research and establish custom rules used to general information processing, make manufacturing and service enterprises more efficiently build a modern information system, and facilitate practical application for Internet of things technology 


\section{System Analysis}

\subsection{Design Aim}

In traditional manufacturing and services industries, the informatization plays an important role for supporting its development, such as bar code technology [4], infrared scanning and RFID technology having been widely used. However, the current extent of information sharing is still a lot of distances away from the level of Internet of things. For example, the bar code of goods is merely passive, which exists only in the process of recording and processing production line, warehouse and sales network, and is still lack of mechanisms for real-time tracking it. If we can effectively introduce mechanisms of Internet of things that can help enterprises grasp the real-time sales situation, combined with production or customer information, location information, transportation and warehouse information, etc., it can help companies more real-time, scientifically arrange procurement, production, storage, distribution, allocation and sales work.

Results of our study will contribute to enhance the informatization extent to traditional manufacturing and services enterprises. If there are many companies to carry out various forms of cooperation, it cannot only help them improve production efficiency, but also gain the good economic benefits for themselves.

\subsection{Demand and Function}

In most existing MIS in manufacturing and services enterprises, data entry and management are mainly by manual inputs or management approach. This mode may result in inefficient management and information lag and other issues. Our study is designed to change the traditional data-management mode in information system by Internet of things, and make information management of enterprises more automatically and intelligent.

Required functions of our MIS are as follow.

1) Information collection, which can non-stop gather various information to ensure manufacturing and services enterprises to perform real-time data management.

2) Intelligent processing algorithms, which can automatically call a specific event that information systems define and achieve intelligent processing to system data.

3) Developing system interfaces, which intelligent processing algorithms can call and implement specific data processing in the system.

\subsection{Conceptual Model}

Combined with the construction model based on Internet of things [5], we have seen a product system that contains sensing, storage, data processing, and decision capabilities at four intelligence levels. Address the above functional requirements, we outline a conceptual model of MIS used to manufacturing and services enterprises, shown in Figure 1, which mainly includes perception layer, control layer and information processing layer three layers.

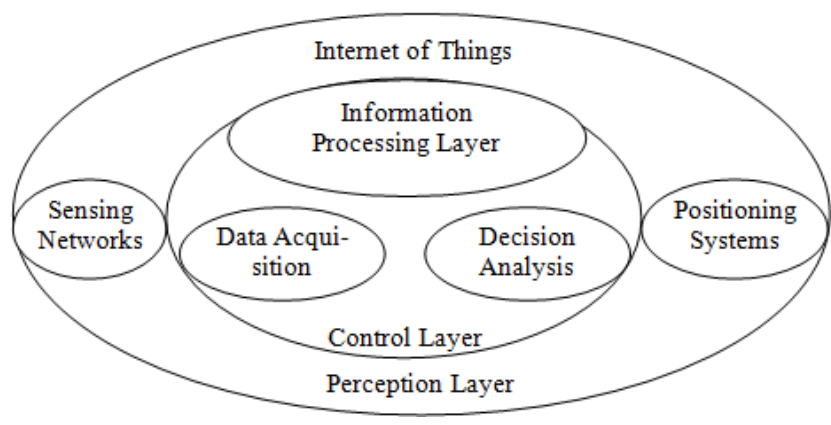

Figure 1. Conceptual model of MIS.

The perception layer is mainly used to sense data, while the control layer is mainly for data processing and information interface call. In the conceptual model, we takes sensing networks in Internet of things and positioning systems as perception layer, which gain relevant entity information such as the coding information and physical location information. The acquired data transports to the control layer of the system through a particular protocol, and the control layer is for data analysis. The latter analyzes subsequent operations that the decision system may request, and calls corresponding interfaces in information processing layer for data processing to ensure the automatic operation of MIS.

\section{System Design}

\subsection{Structure Design}

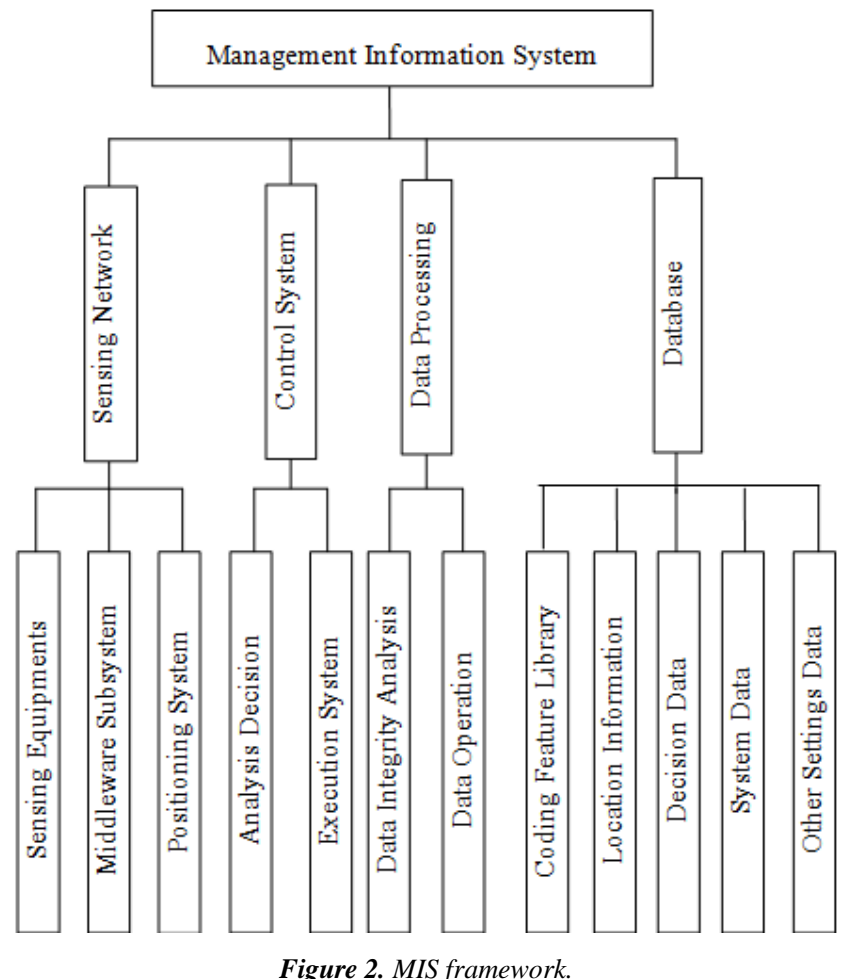

MIS is mainly used to automatically and intelligently process data from manufacturing and services enterprises, while the database as data warehouse is also bound to be a key part of it. Combined systematic conceptual model presented in 
Figure 1, we construct an MIS framework of manufacturing and service enterprise, shown in Figure 2.

MIS is composed of sensing network, control system, data processing module and database four parts, and its composition and function of each part is as follows.

\subsubsection{Sensing Networks}

It is primarily responsible for gathering entity information in manufacturing and services enterprises such as the feature and the physical location, and this part is mainly composed of information sensing equipment, intelligent signal processing and smart positioning system.

1) Signal sensing equipments are mainly responsible for obtaining enterprise entities' unique encoding or characteristics.

2) RFID middleware subsystem is mainly responsible for intelligent signal processing to convert and unify data format and interpret specific information used to the required operation. We will explain in detail this subsystem in section 4 .

3) Positioning system is mainly responsible for gaining enterprise entities' physical location information, which will be stored in the database later.

\subsubsection{Control System [6]}

It can analyze and make decision by calling information system interface, and it is mainly composed of decision system and execution system. Decision system is as a core connected to sensing networks, which includes sensing-data acquisition module, data comparison and analysis module as well as sensing-network control module. Execution system includes enterprise-information interface calling module, information analysis and execution module. The components and functions of the two systems are as follows.

1) Sensing data module is mainly used to obtain sensing data received from sensing networks.

2) Data analysis module is mainly responsible for analyzing the sensing data from networks, comparing their feature information and location information with the same items in background database, so as to find the corresponding information system interface to perform system call.

3) Interface call module is mainly responsible for calling information interface and saving sensing data to the appropriate module of information system.

4) Data tracking module is mainly responsible for analyzing completeness and accuracy of sensing data, returning data entry to the decision system, and then control-sensing network starts a new round of data collection.

\subsubsection{Data Processing Module}

This module is mainly responsible for analyzing the data coming from the interface and performing informatization processing, which includes data integrity analysis and the operation such as data format check, data modify, data add or delete.

Database: it is mainly responsible for the data storage and retrieval operations. Stored information includes coding library and feature library, location information, analysis and decision data, system data, and other settings parameters.
Functions of each part are as follows.

1) Coding library and feature library are used to establish enterprises entity information. A unique entity code represents an individual entity, firstly written into coding library. Subsequently, the other information such as entity name, entity material and entity size, is also written into feature library. By querying entity code, it can obtain its feature information.

2) Location information is mainly used to save position-encoding information of each entity, by which people can obtain entity location name through the position encoder. The location name is usually corresponding to a work phase or other specific meaning.

3) Analysis and decision data mainly saves interface information that is sourced to entity code and entity location, and provides analysis data for decision systems.

4) System data is mainly used for system data storage.

5) Other parameters setup is used for setting parameters for other module in MIS.

\subsection{System Running}

Only when mutual coordination and mutual convergence among the various modules in MIS exists, it can ensure information system to work effectively and stably. System running procedure is as shown in Figure 3.

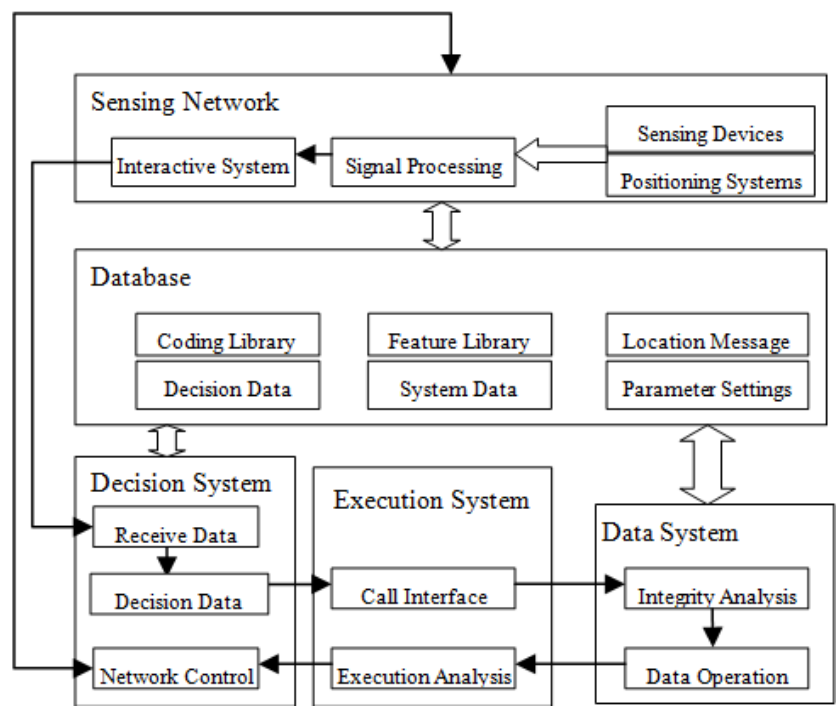

Figure 3. System running procedure.

\subsubsection{Sensing Networks}

It collects business entity information, which includes unique encoding or features message and location coding, by information-sensing devices and smart positioning systems.

And then it performs intelligent processing to provide data that the analysis and decision system can identify. Subsequently, the sensing networks through intelligent interactive system deliver sensing data to the decision system.

\subsubsection{Decision System [7]}

It receives business entities' messages coming from sensing network, including the only coding information or features 
and physical location information, and then executes data analysis.

On the one hand, by comparing the analysis and decision data in database, it can find the informatization interfaces that the system will pre-call; on the other hand, information system can find out correspondingly specific meaning by querying entity physical location or unique encoding from the database, such as a name corresponding to a working stage. Analysis and decision system will finally transfer the decision data to the execution system.

\subsubsection{Execution System}

It receives data from the decision system, calls corresponding interfaces in MIS, and transmits sensing data to the data system interface.

\subsubsection{Data System [8]}

It is called and then executes the corresponding informatization operations. First, data integrity analysis should be done to ensure the accuracy of sensing data, then performs the corresponding database operation and feedback, and output the results. By control system and decision system, the further action in sensing network will be controlled better so as to ensure the repeatedly effective operation of MIS.

During intelligent processing information, specific data stored in the database plays a fundamental role, for example, calling up information interface from the database according to entity information and its physical location information. This information is the predefined parameter stored in the database when information system is established. Data will be timely read as the system is running. As long as there are changes in enterprise business, it will be modified.

Effective operation of information systems mostly depends on accurately obtaining the entity information and physical location information. The analysis and decision system decide to call which information interface, and the execution system by interface call ensures the effective operation of the system.

\section{RFID Middleware Subsystem}

As the nerve center of Internet of things, RFID middleware can connect tag readers and enterprise application, eliminating the complexity maintenance, reducing the cost of enterprise integration. However, RFID middleware is also a complex and important system, needing further promote the use, progressively improve and perfect.

RFID middleware subsystems in our framework model shown in Figure 1 are mainly responsible for data format conversion in sensing network, which is essentially a service program that directly interacts with tag reader device. Its main functions include the RFID reader data extraction, filtering, processing and integration with other application interfaces, and the application framework is as shown in Figure 4.

In Figure 4, the imaginary part of the coil is the RFID middleware. Reading continuously a large number of tags, the RFID reader transfers data to RFID middleware to process. RFID as a the key technology is also used in the field of manufacturing, assembly, postal services, warehousing and asset management, to use a smart RFID middleware system will make these applications become more convenient and efficient.

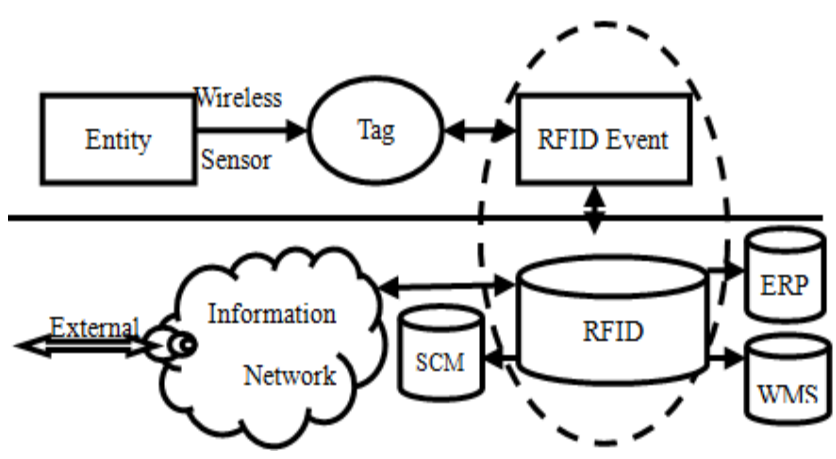

Figure 4. Application framework of RFID middleware.

\section{Experimental Scheme}

Our designed MIS can be tested on J2EE platform [9], using a combination of Struts, Spring, Hibernate, Servlet, HTML, JavaScript and other technology to develop a software and prepare for its application to logistics company.

\subsection{System Construction}

1) Sensing networks

This module uses common collection devices with microcontroller to gather information. Processing algorithms integrated in the device chip is used to complete the intelligent processing during information acquisition. The only identification and other information that is collected from sensing networks every time, will store in the temporary table of database, and analysis and decision system obtains data source by reading the data in the temporary table.

2) Decision system

Through the Servlet technology and database technology, analysis and decision system is built, which defines the interaction with the sensing network Servlet, and our MIS takes .listener suffix as a request that is interpreted and executed by analysis and decision systems.

3) Execution system

The execution system mainly defines extensive interface of information systems and analyzes the executed results of MIS, and it uses the way of Java class to be defined and implemented.

4) Data system

This module can use Struts + Spring + Hibernate framework for developing, adopting MVC [10] control mode, in accordance with the typical three-layer structure, similar with existing information systems in development mode. Data systems, analysis and decision system, execution system together form a software platform of MIS. Analysis and decision system and execution systems as a connecting bridge between data systems and sensing networks also are an important guarantee to implement MIS automated and intelligent. 


\subsection{Application Results}

We apply our design scheme to a logistics company for detecting the goods out of storage, shown in Figure 5.

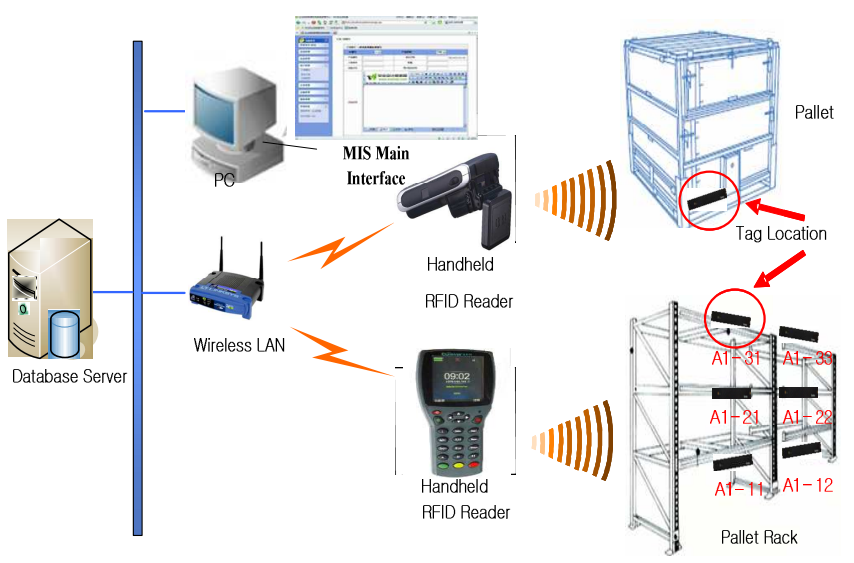

Figure 5. Detection to goods out of storage.

Practical results show that our MIS can display the physical location of goods in real time, all the information on the items can be collected and tracked in real time, and provide a strong support to goods out of storage for its effective management and control.

\section{Conclusions}

This paper systematically talks about integrating, processing and converting data, fully connects the physical world to the digital world to complete the accurate mapping between the two part, for the manufacturing and service enterprises to provide an enterprise-class product development scheme. The MIS model we present based on Internet of things for manufacturing and service enterprises, can make a complex product-development system more clearly, dynamically and completely controlled in the hands of managers. Thus, quality and reliability of the manufacture products or services has a systemic guarantee, contributing to implement the production and management informatization, and its productivity will have greatly improved, too.

From the application level, on this basis of the conceptual model of management information system based on Internet of things, people can further research to establish general information processing custom rules, and enhance constructing efficiency for MIS. This is certainly conducive to the wide promote of Internet of things technology and its applications.

\section{Acknowledgements}

The research was supported by Artificial Intelligence Key Laboratory of Sichuan Province (No. 2013RYY04) and the Sichuan Provincial Education Department's Key Project (No.14ZA0210).

Our work was also supported by university Key Laboratory of Sichuan Province (No. 2013WYY09) and Fund Project of Sichuan Provincial Academician (Experts) Workstation (No.2014YSGZZ02).

\section{References}

[1] Yuan J S, Hu Y. Implementation of RFID Middleware Based on Hash Chain[J]. Applied Mechanics and Materials, 2013:12-15.

[2] Ghonaim W, Ghenniwa H, Shen W. Towards an agent oriented smart manufacturing system[C]. //Computer Supported Cooperative Work in Design (CSCWD), 2011 15th International Conference on. IEEE, 2011:636 - 642.

[3] Kopetz H. Real-Time Systems[M]. Springer US, 2011:307-323.

[4] Li D, Wei Q, Liu C, et al. A novel verification code technology to make web system more secure[C]. //Computer Science \& Education (ICCSE), 2011 6th International Conference on. IEEE, 2011:1303 - 1306.

[5] Kiritsis D. Closed-loop PLM for intelligent products in the era of the Internet of things[J]. Computer-Aided Design, 2011, 43(5): 479-501.

[6] Ellis G. Control system design guide: using your computer to understand and diagnose feedback controllers[M]. Butterworth-Heinemann, 2012.

[7] Ostwald D, Porcaro C, Mayhew S D, et al. EEG-fMRI based information theoretic characterization of the human perceptual decision system[J]. PloS one, 2012, 7(4): e33896.

[8] Foster I, Vockler J, Wilde M, et al. Chimera: A virtual data system for representing, querying, and automating data derivation $[\mathrm{C}] / /$ Scientific and Statistical Database Management, 2002. Proceedings. 14th International Conference on. IEEE, 2002: 37-46.

[9] $\mathrm{Qu} \mathrm{C}$, Engel $\mathrm{T}$, Meinel C. Implementation of an enterprise-level groupware system based on J2EE platform and WebDAV protocol[C]. //Enterprise Distributed Object Computing Conference, 2000. EDOC 2000. Proceedings. Fourth International. IEEE, 2000:160 - 169.

[10] Lin Y J, Lin S. Research on E-Commerce Software Framework of MVC Mode Based on .NET[J]. Applied Mechanics and Materials, 2013:2645-2648. 